\title{
Analysis of Surface Acoustic Wave Propagation Velocity in Biological Function-Oriented Odor Sensor
}

\author{
Yuxia Yang $\mathbb{D}^{1}{ }^{1}$ Koji Nagano $\mathbb{D},{ }^{2}$ Chaoluomeng $\mathbb{D},{ }^{2,3}$ Tatsuo Iwasa $\mathbb{D}{ }^{2}$ \\ and Hisashi Fukuda $\mathbb{( D}^{2}$ \\ ${ }^{1}$ Division of Production and Information Systems Engineering, Muroran Institute of Technology, Muroran 050-8585, Japan \\ ${ }^{2}$ Division of Engineering, Muroran Institute of Technology, Muroran 050-8585, Japan \\ ${ }^{3}$ College of Animal Science and Technology, Inner Mongolia University for Nationalities, Tongliao 028000, China
}

Correspondence should be addressed to Yuxia Yang; 13092501@mmm.muroran-it.ac.jp

Received 29 August 2017; Revised 22 November 2017; Accepted 17 December 2017; Published 13 March 2018

Academic Editor: Stephane Evoy

Copyright (c) 2018 Yuxia Yang et al. This is an open access article distributed under the Creative Commons Attribution License, which permits unrestricted use, distribution, and reproduction in any medium, provided the original work is properly cited.

\begin{abstract}
We describe the measurement of surface acoustic wave (SAW) velocities to identify five odorant molecules by using a SAW device system. We derive a new frequency equation for SAWs propagating in the SAW device system with Cynops pyrrhogaster lipocalin (Cp-Lip1) protein, in order to identify five odorant molecules, R-limonene (R-Lim), ethyl butyrate (Eth), 2-isobutylthiazole (Iso), benzophenone (Ben), and 2-acetylthiazole (Ace). We developed a method to identify these odorant molecules combined with the Cp-Lip1 odorant-binding protein. Our frequency equation can satisfactorily predict different odorant molecules in the Cp-Lip 1 SAW device. Moreover, our data suggest that the propagation velocity of the SAWs mostly relate to the density and concentration of the Cp-Lip1 odorant molecule mixtures. At the same sample concentration, the propagation velocity depends on the density. For the same odorant molecule, the propagation velocity decreases with increasing concentration.
\end{abstract}

\section{Introduction}

In the past few decades, odor sensors oriented toward biological functions have attracted considerable interest. A device known as an odor sensor is usually a multisensor system based on some sensing principle, and a single sensor is used to display drift and lack of sensitivity. This results in obvious defects in calibration and reproducibility of the sensor array. The biological function-oriented odor sensor combines the sensing technology and the biological olfactory principle to improve the artificial recognition of the smell and thus closer to the good performance of the biological olfaction. Biological function-oriented odor sensors with sensor-based noses are required to fulfill the needs of various applications of the biological electronic nose systems. In some instances, biological function-oriented odor sensors can be used in medical applications, biochemical research, and environmental monitoring. Electrochemical methods, optical methods, and acoustic techniques are used in biological function-oriented odor sensors [1]. Among these methods, acoustic techniques have lately received considerable attention for odor sensor systems because of their high sensitivity, rapid detection, and safety [2-4]. Surface acoustic wave (SAW) devices, in which Rayleigh waves propagate, have also been studied for biological function-oriented odor sensors [5].

However, SAW device sensors with odorant medium solutions have certain drawbacks, and it is worth investing effort into addressing these drawbacks. The development of biological function-oriented odor sensors is an ongoing challenge owing to the hydrophobic nature of odorant molecules [6,7]. Experimental investigations on odorant molecules have shown that olfactory receptors can directly bind with odorant molecules, but most odorant molecules are low-molecular compounds that are volatile and hydrophobic [8-11]. However, although some promising progress has been made through these experiments, further theoretical research on SAW device sensors is still required [12-14].

Among various odor sensors, SAW device sensors have emerged as an interesting candidate because of their ability to concentrate wave energy on the piezoelectric material 
surface [5]. In particular, the high-frequency applications for SAW device sensors are in the range of $100 \mathrm{MHz}$ to a few GHz [4]. SAW devices have also been used as chemical sensors for gas detection [15-17]. They have therefore been targeted for use as biological function-oriented odor sensors.

To overcome the issues due to the hydrophobic nature of odorant molecules, we thought that it is extremely important to use odorant-binding proteins with the SAW device sensors. Odorant-binding proteins are water-soluble small protein, which can bind and solubilize odorants in the aqueous environment [11]. Although a few studies have reported the use of odorant-binding protein $[18,19]$ or the peptide based on the odorant-binding protein [20], to the best of our knowledge, no report was found that has reported the application of SAW device sensors based on odorant-binding proteins except for only one symposium report [21]. We use Cynops pyrrhogaster lipocalin (CpLip1) protein, an odorant-binding protein discovered in newts, which can combine with various hydrophobic odorant molecules [11].

The Cp-Lip1 SAW device system refers to different odorant molecules bound to the Cp-Lip1 odorant-binding protein in a SAW device. To verify the validity of the CpLip1 SAW device system as a biological function-oriented odor sensor, we experimentally observed the propagation velocity of the SAW device with different odorant molecules bound to the Cp-Lip1 protein. We also theoretically calculated the propagation velocity of surface acoustic waves propagating in the SAW device. There are two general modes of SAWs, that is, Rayleigh wave and leaky Rayleigh wave, propagating in SAW devices [5]. The frequency equation of the Rayleigh wave was calculated in 1960 [22]. Since then, many studies have also focused on the leaky Rayleigh wave [23]. However, those studies did not provide the frequency equation of the leaky Rayleigh wave. Therefore, here, we derive the frequency equation of the leaky Rayleigh wave based on the elastic wave propagation theory. The leaky Rayleigh wave radiates as a longitudinal wave into a liquid [23]. Thus, the boundary conditions of the liquid-solid interface were theoretically expounded in this paper. Then, experimental results of the Cp-Lip1 odorant mixture were compared with the corresponding theoretical results and analysed.

For theoretically calculating the propagation velocity, it is difficult to precisely define the total density of the odorant medium. To overcome this problem, we adopt the total mass and total volume in the calculations. The crucial aspect of calculating the total mass is the Cp-Lip1 odorant mixture mass. Moreover, the propagation velocity of the longitudinal wave of the liquid should also be calculated. We solve these problems by calculating the total volume of the odorant medium.

This paper is organized as follows. In Section 2, we derive the frequency equation of the surface acoustic wave velocities propagating in the SAW device sensor. And, we observe the SAWs in a SAW device in which Cp-Lip1 odorant mixture is used as the liquid medium. We then analyse the experimental results of the Cp-Lip1 SAW

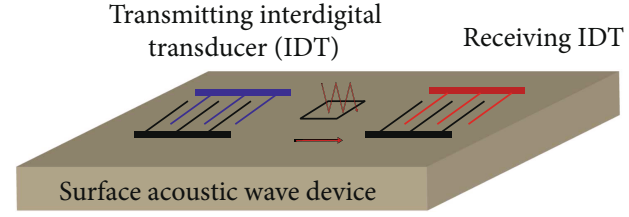

Figure 1: Cp-Lip1 SAW device.

TABLE 1: Five odorant molecules used in the measurement experiments.

\begin{tabular}{lccc}
\hline $\begin{array}{l}\text { Odorant } \\
\text { molecules }\end{array}$ & Chemical name & $\begin{array}{c}\text { Molecular } \\
\text { weight }\end{array}$ & $\begin{array}{c}\text { Density } \\
\left(\mathrm{g} / \mathrm{cm}^{3}\right)\end{array}$ \\
\hline R-Lim & R-Limonene & 136.24 & 0.840 \\
Eth & Ethyl butyrate & 116.16 & 0.879 \\
Iso & 2-Isobutylthiazole & 141.23 & 1.020 \\
Ben & Benzophenone & 182.22 & 1.111 \\
Ace & 2-Acetylthiazole & 127.16 & 1.227 \\
\hline
\end{tabular}

device system. Section 3 presents a comparison between the theoretical and experimental results, and finally, in Section 4, we discuss these results and present the conclusions of this study.

\section{Materials and Methods}

2.1. Materials. In this paper, we considered a Cp-Lip1 SAW device system to identify five odorant molecules. This system was based on the Rayleigh-type SAW device developed by the semiconductor device research laboratory from the Muroran Institute of Technology [5]. We measured different kinds of Cp-Lip1 odorant mixture on the Rayleigh-type SAW devices. Therefore, this device was referred to as the Cp-Lip1 SAW device, and it is shown in Figure 1. The Cp-Lip1 SAW device system has used a shear horizontal SAW (SH-SAW) mode. An interdigital transducer (IDT) can process electric signals to SAWs via the piezoelectric effect [5]. We used a Cp-Lip1 SAW device whose frequency is $157.6 \mathrm{MHz}$. The piezoelectric substrate layer is $42 \mathrm{Y}-45 \mathrm{X}$ ST-cut quartz. The distance between the two IDT electrodes was $25.00 \mathrm{~mm}$. Five Cp-Lip1 odorant mixtures were placed in a groove on the Cp-Lip1 SAW devices. The width and height of the groove were $0.41 \mathrm{~mm}$ and $0.26 \mathrm{~mm}$, respectively.

To detect the odorant by the SAW devices, the velocity change due to the odorant-binding should be measured. As the first step of the study, the Cp-Lip1 and odorant were premixed to form the Cp-Lip1 odorant mixtures, and the (velocity of the Cp-Lip1 odorant mixtures) change due to the odorant-binding was measured by the velocity difference between the Rayleigh wave and the total velocity of the surface acoustic waves when the Cp-Lip1 odorant mixtures are in the groove. Table 1 presents the five odorant molecules used in the experiments. These odorant molecules bind to the Cp-Lip1 protein with high affinity [24]. The molecular weight of the Cp-Lip1 protein was estimated to be 18604.56, which is much higher than that of the 
odorant molecules. The sample solutions, the Cp-Lip1 and odorant mixtures, were prepared by binding the Cp-Lip1 and one of the five odorant molecules, which were used in this report to test the Cp-Lip1 SAW device. In addition, a tris(hydroxymethyl)aminomethane or THAM buffer was used to stabilize the $\mathrm{pH}$ of the odorant medium. The molecular weight of the tris base was 121.14, and the density was $1.353 \mathrm{~g} / \mathrm{cm}^{3}$. These five sample solutions were used to analyse the surface acoustic wave velocity of the Cp-Lip1 SAW device.

2.2. Methods. The surface acoustic wave velocity due to Cp-Lip1 SAW device calculation formula was derived. We then measured the experimental velocity of the SAW by using the Cp-Lip1 SAW device system. As it is well known, some studies have analyzed the isotropic models $[25,26]$. However, they do not directly calculate the propagation velocity of elastic waves by using the density of the liquid and Poisson's ratio of the substrate material. Figure 2 shows the SAWs propagating in the Cp-Lip1 SAW device and the propagation distance of the SAW. When the liquid is on the quartz substrate, the SAW propagates from the IDT generating leaky Rayleigh waves. The wave travels as a radiating longitudinal wave into the liquid. The radiation angle of the longitudinal wave at the liquid-solid interface is the leaky Rayleigh angle, and it is denoted as $\theta_{\mathrm{LR}}$ in this paper. If the groove is loaded with a liquid, the leaky Rayleigh waves are excited by the wave incident from the coupling liquid. The wave energy is lost by irradiation into the liquid due to coupling interaction. The coupling effects of the interaction between liquid and solid interface also affects the propagation velocity of surface acoustic waves.

Consider a point $\mathrm{P}(x, y$, and $z)$ in an isotropic solid and hypothesize an ideal solid. The density of the solid material is $\rho_{s}$, and an isotropic solid has only two elastic constants, that is, Lame's constants, $\lambda$ and $\mu$, wherein $\mu$ is also called the rigidity or shear modulus [22]. Suppose that point $\mathrm{P}$ is displaced, with the coordinates of the displacement being $(u, v$, and $w)$. The equations of motion are not supported by the transverse wave for an ideal liquid. These equations will be satisfied if the potential functions $\phi$ and $\varphi_{i}(i=x, y, z)$ are solutions of the wave equations. The wave equations are

$$
\begin{aligned}
\nabla^{2} \phi & =\frac{1}{c_{d}^{2}} \cdot \frac{\partial^{2} \phi}{\partial t^{2}}, \\
\nabla^{2} \varphi_{i} & =\frac{1}{c_{t}^{2}} \cdot \frac{\partial^{2} \varphi_{i}}{\partial t^{2}},
\end{aligned}
$$

where $c_{d}$ and $c_{t}$ are the velocities of the longitudinal wave and transverse wave, respectively, in the substrate of the Cp-Lip1 SAW device. The boundary conditions of the liquid-solid interface are that the pressure in the liquid is equal to the normal stress in the solid, and the transverse stress is zero [22]. To derive the equations describing the surface acoustic wave motion in a Cp-Lip1 SAW device, we again consider

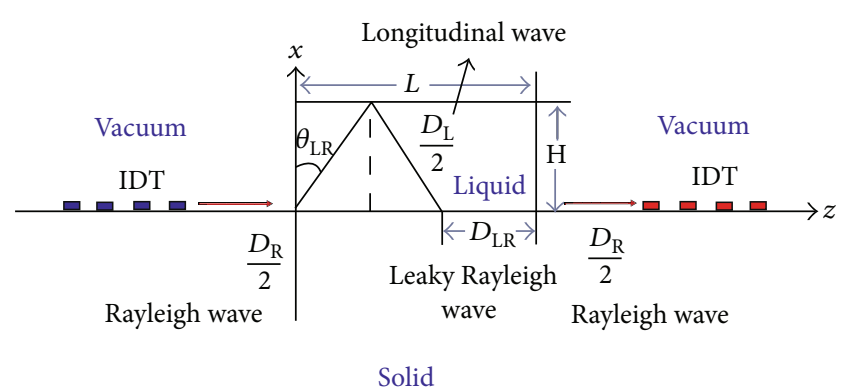

FIgURE 2: Surface acoustic waves propagating in the Cp-Lip1 SAW device and propagation distance of SAW.

the equilibrium of a volume element. Then, we have three equations of $\rho_{s}$ and $x, y$, and $z$ directions on the surface facing the positive coordinate direction. These equations,

$$
\begin{gathered}
\rho_{s} \frac{\partial^{2} u}{\partial t^{2}}=\frac{\partial \tau_{x x}}{\partial x}+\frac{\partial \tau_{y x}}{\partial y}+\frac{\partial \tau_{z x}}{\partial z} \\
\rho_{s} \frac{\partial^{2} u}{\partial t^{2}}=\frac{\partial \tau_{x y}}{\partial x}+\frac{\partial \tau_{y y}}{\partial y}+\frac{\partial \tau_{z y}}{\partial z} \\
\rho_{s} \frac{\partial^{2} w}{\partial t^{2}}=\frac{\partial \tau_{x z}}{\partial x}+\frac{\partial \tau_{y z}}{\partial y} \times \frac{\partial \tau_{z z}}{\partial z}
\end{gathered}
$$

are true for both isotropic and anisotropic materials. We insert those boundary conditions into the potential functions and calculate the frequency equation. Assume that the propagation velocity of the leaky Rayleigh wave is $c_{\mathrm{LR}}$ and the velocity of the longitudinal wave in the liquid medium is $c_{\mathrm{L}}$. The frequency equation of the Cp-Lip1 SAW device is

$$
\begin{aligned}
8\left(\frac{c_{t}}{c_{\mathrm{LR}}}\right)^{2}\left\{1-\left(\frac{c_{t}}{c_{\mathrm{LR}}}\right)^{2}\right\} \\
\cdot\left\{2\left(\frac{c_{t}}{c_{\mathrm{LR}}}\right)^{2}\left(\frac{c_{t}}{c_{d}}\right)^{2}-2\left(\frac{c_{t}}{c_{\mathrm{LR}}}\right)^{2}+1\right\}-1 \\
=-j\left(\frac{\rho_{l}}{\rho_{s}}\right) \frac{\left(c_{t} / c_{\mathrm{LR}}\right)^{2}-\left(c_{t} / c_{d}\right)^{2}}{\left(c_{t} / c_{\mathrm{L}}\right)^{2}-\left(c_{t} / c_{\mathrm{LR}}\right)^{2}}
\end{aligned}
$$

where $j$ is a complex number. The relationship between Poisson's ratio $(v)$ of substrate of Cp-Lip1 SAW and $\left(c_{t} / c_{d}\right)^{2}$ is

$$
\left(\frac{c_{t}}{c_{d}}\right)^{2}=\frac{1-2 v}{(2-2 v)},
$$

where $c_{d}$ and $c_{t}$ are the velocities of the longitudinal wave and transverse wave, respectively, in the substrate of the Cp-Lip1 SAW device. In (3), when $\rho_{l}$ is zero, the right side of the equation becomes zero. In other words, it becomes the frequency equation of the Cp-Lip1 SAW device at the vacuum-solid interface. This surface wave is known as the Rayleigh wave [22]. Also, it was observed that (3) has eight complex roots and two real roots. The pair of real roots shows the propagation velocity of the leaky Rayleigh waves. 
The previously mentioned $\theta_{\mathrm{LR}}$ depends on $c_{t}$ and $c_{\mathrm{L}}$. By Snell's law, we have

$$
\left(\frac{c_{t}}{c_{\mathrm{L}}}\right)=\frac{1}{\sin \theta_{\mathrm{LR}}} .
$$

We observed that (3) can be used to theoretically calculate the propagation velocity of surface acoustic waves of the Cp-Lip1 SAW device. However, even more importantly, it should clarify the density of the Cp-Lip1 odorant mixture. The volume and mass of the odorant medium were used for these calculations.

The propagation distance of the SAWs in the Cp-Lip1 SAW device is shown in Figure 2. As it can be seen, the geometric relationships are

$$
\begin{aligned}
D_{\mathrm{LR}} & =L-2 H \tan \theta_{\mathrm{LR}}, \\
D_{\mathrm{L}} & =\frac{2 H}{\cos \theta_{\mathrm{LR}}},
\end{aligned}
$$

where the width and height of the groove are $L$ and $H$, respectively. The propagation distance of the Rayleigh wave, leaky Rayleigh wave, and longitudinal was $D_{\mathrm{R}}, D_{\mathrm{LR}}$, and $D_{\mathrm{L}}$, respectively. The propagation velocity of the liquid $c_{\text {Liquid }}$ depends on the propagation velocities of the leaky Rayleigh wave and longitudinal wave. Hence, it is calculated by

$$
c_{\text {Liquid }}=\frac{D_{\mathrm{L}}}{D_{\mathrm{R}}+D_{\mathrm{L}}+D_{\mathrm{LR}}} \cdot C_{\mathrm{L}}+\frac{D_{\mathrm{LR}}}{D_{\mathrm{R}}+D_{\mathrm{L}}+D_{\mathrm{LR}}} \cdot C_{\mathrm{LR}} \text {. }
$$

Our theory was verified through experimental studies. The propagation time of the SAWs was measured by the Cp-Lip1 SAW device. The Rayleigh wave propagates on a free surface. Thus, it was measured in the Cp-Lip1 SAW device without a groove. The propagation time of the Rayleigh wave can be calculated as an interval between the input and output signals by analysing the cross-correlation coefficients. The peak of the cross-correlation corresponds to the interval. This interval is regarded as the propagation time of the Rayleigh wave. The leaky Rayleigh wave and longitudinal wave also propagate on the Cp-Lip1 SAW device when the liquid is in the groove. The velocity of the Cp-Lip1 odorant mixtures can be measured by the velocity difference between the Rayleigh wave and the total velocity of the surface acoustic waves when the Cp-Lip1 odorant mixtures are in the groove.

Pure water was used as the standard solution. $10 \mu \mathrm{M}$ of the Cp-Lip1 protein in $10 \mu \mathrm{M}$ Tris buffer was used as the binding solution. The sample solution was prepared by binding the Cp-Lip1 binding solutions and five odorant molecules, and it was used to identify the different odorant molecules in the Cp-Lip1 SAW device. Odorants, R-Lim, Eth, Iso, Ben, and Ace were used at $0.1 \mathrm{mM}$ or $1 \mathrm{mM}$ in this experiment. Figure 3 shows the measurement setup of the propagation velocity of SAWs with the Cp-Lip1 odorant mixtures in the Cp-Lip1 SAW device. The standard solutions and sample solutions were placed in the groove. We

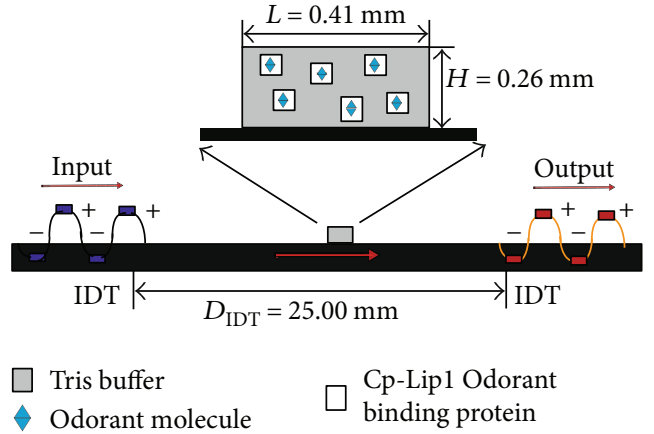

FIGURE 3: Measurement of propagation velocity of SAWs with Cp-Lip1 odorant mixture in the Cp-Lip1 SAW device.

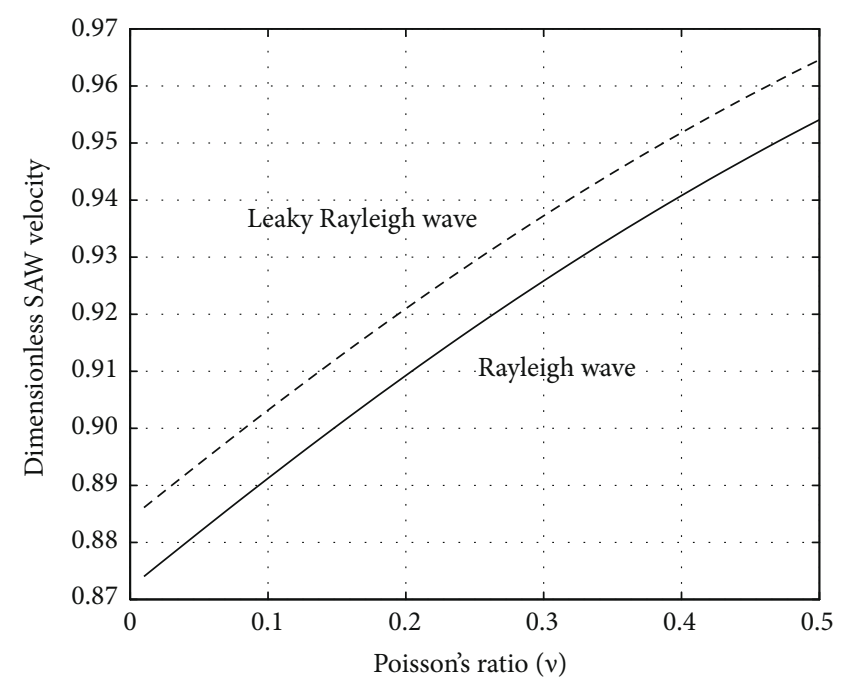

FIgURE 4: Dimensionless velocity of Rayleigh wave and leaky Rayleigh wave in different materials.

measured waves 30 times in each solution. Details of the signal processing method are as follows: (1) synchronously inputting signals while determining the corresponding output signals; (2) synchronously adding signals to determine the exact measurement signal; (3) calculating signal delay time by analysing cross-correlation coefficients; (4) calculating the propagation velocity of the SAW based on the delay time; (5) and calculating the standard deviation based on the signals measured 30 times.

\section{Results}

Figure 4 shows the relationship between Poisson's ratio and the dimensionless SAW velocity. The dotted and solid lines in the figure show the propagation velocity of the leaky Rayleigh wave and Rayleigh wave, respectively. Theoretical results show that the dimensionless velocity of the leaky Rayleigh wave was faster than that of the Rayleigh wave in the same substrate. The results also agree with those from previous research on Rayleigh waves [22]. This also proves 


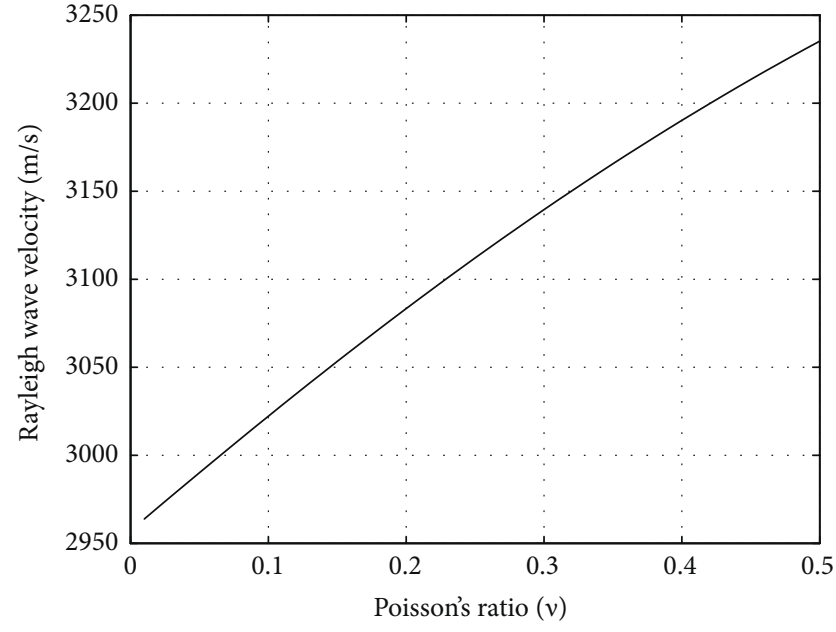

FIGURE 5: Propagation velocity of Rayleigh wave in different materials.
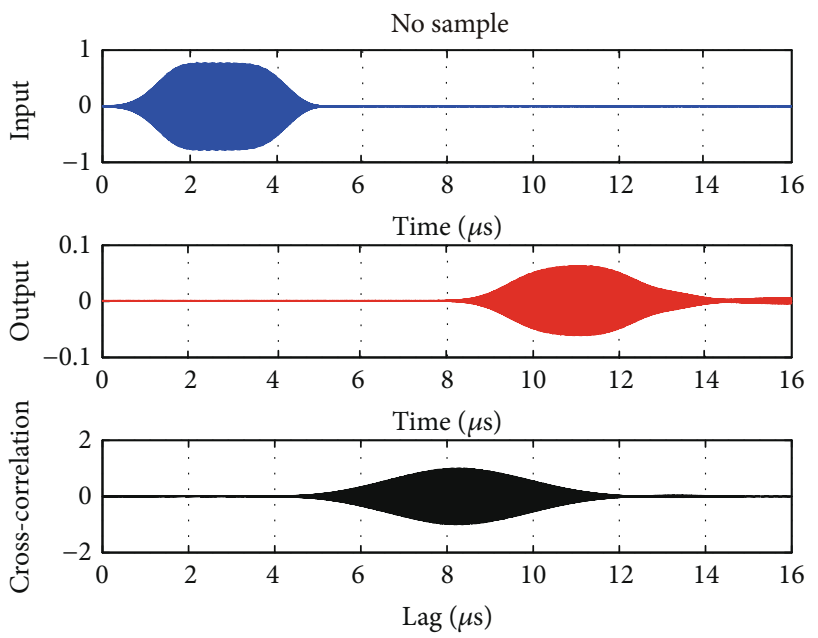

FIGURE 6: Measurement signals of no sample in the Cp-Lip1 SAW device and cross-correlation coefficient.

that our theoretical results are significantly reliable. Further, the theoretical velocity of the Rayleigh wave was calculated by (3). Figure 5 shows the propagation velocity of the Rayleigh wave in different materials. Poisson's ratio of quartz used in our experiment is 0.266 . The theoretical calculation results show that the propagation velocity of the Rayleigh wave was $3118 \mathrm{~m} / \mathrm{s}$ in the quartz substrate. In order to confirm this result, we measured the Rayleigh waves in the Cp-Lip1 SAW device without the groove. Figure 6 shows the measurement signals when no sample is present in the Cp-Lip1 SAW device and the cross-correlation coefficient. The experimental data show that the delay time of the input and output signal was $8.235 \mu \mathrm{s}$. As a result, the experimental velocity of the Rayleigh wave was $3036 \mathrm{~m} / \mathrm{s}$. This result agrees very closely with the theoretical calculation result. It shows that the theoretical results can help us in identifying different odorant molecules.

We carried out an analysis to identify the differences between different odorant molecules. For this, the standard
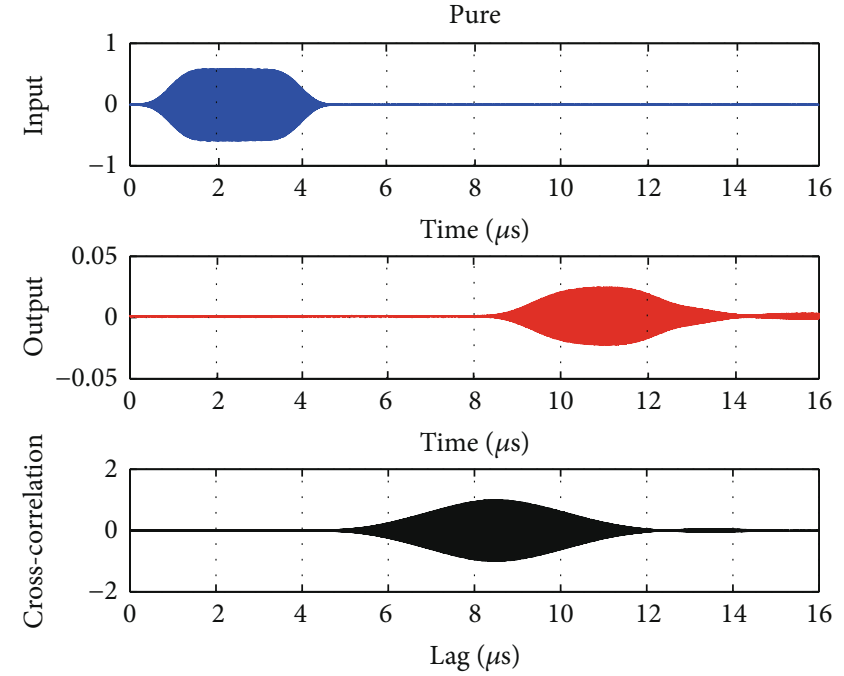

FIgURE 7: Measurement signals of standard solution in the Cp-Lip1 SAW device and cross-correlation coefficient.
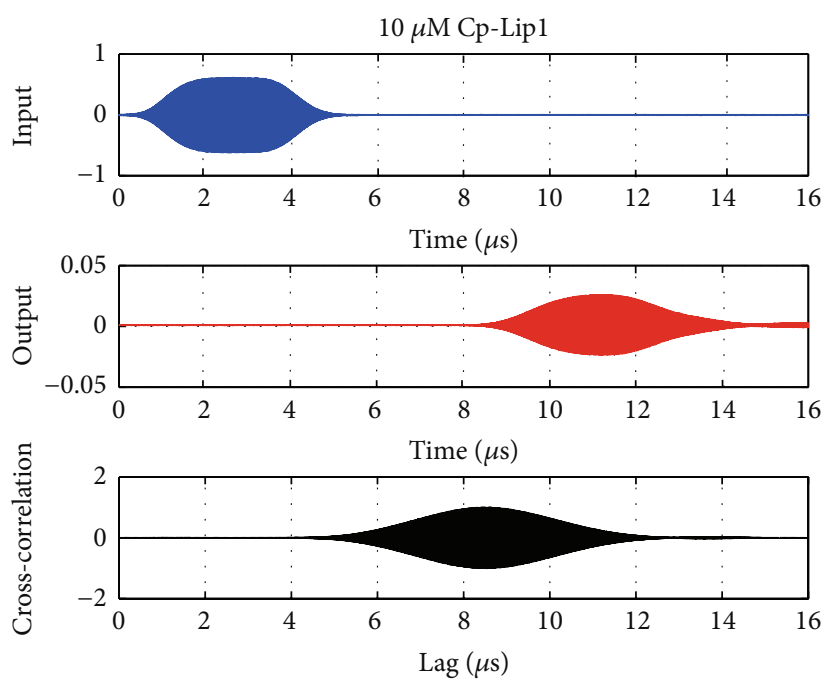

FIgURE 8: Measurement signals of the binding solution $(10 \mu \mathrm{M}$ Cp-Lip1 protein in $10 \mu \mathrm{M}$ Tris buffer) in the Cp-Lip1 SAW device and cross-correlation coefficient.

solution and sample solution were compared in the CpLip 1 SAW device. Figures 7 and 8 show the measurement signals of the standard and the binding solution $(10 \mu \mathrm{M} \mathrm{Cp}-$ Lip1 protein in $10 \mu \mathrm{M}$ Tris buffer) of the Cp-Lip1 SAW device and the cross-correlation coefficient, respectively. In essence, the delay time of the input and output signals was a tabbed time with liquids for groups of propagation time, which contain the propagation time of the leaky Rayleigh wave, longitudinal wave, and Rayleigh wave. Thus, the propagation velocity of the liquid was calculated by using (7). The computed results show that the standard solution velocity was $2068 \mathrm{~m} / \mathrm{s}$, and the velocity for the binding solution was $1168 \mathrm{~m} / \mathrm{s}$.

We then compared the experimental and theoretical results of the sample solutions. Figure 9 shows the SAW 


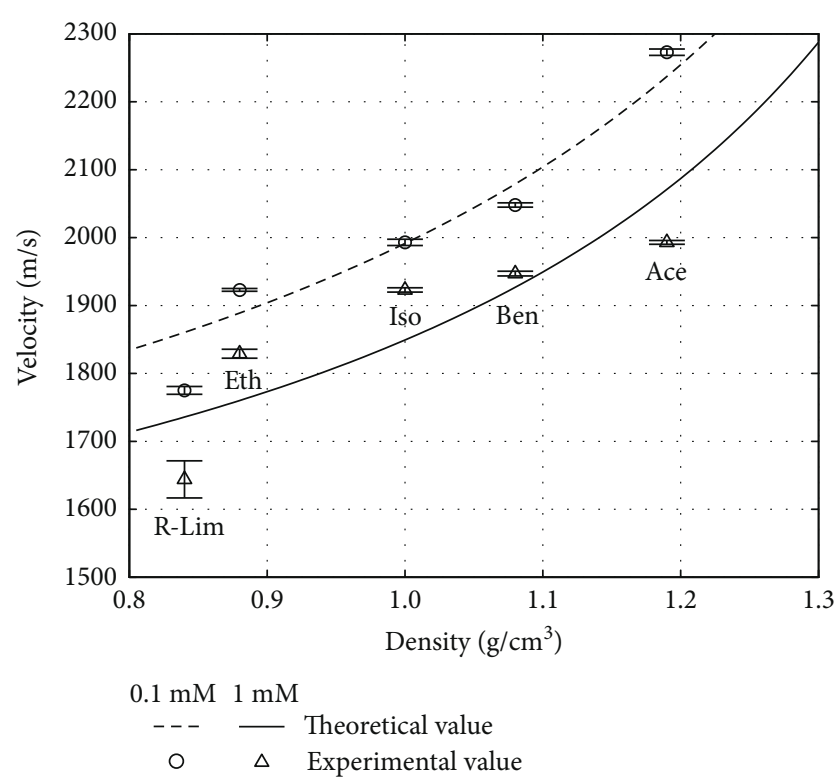

FIGURE 9: SAW velocity of five sample solutions in the Cp-Lip1 SAW device.

velocity of five sample solutions in the Cp-Lip1 SAW device. The experimental results for the Cp-Lip1 binding solutions were in good agreement with the theoretical results, which demonstrate that the frequency equation of the Cp-Lip1 SAW device can successfully identify the odorant molecules in the Cp-Lip1 SAW device. When the concentration is the same, the propagation velocity of the sample solution depends on the density. When the density increases, the propagation velocity also increases. The results illustrate that different odorant molecules in same concentration can be identified because the propagation velocity and density are linearly related in the Cp-Lip1 SAW device. At different concentrations, the propagation velocity at $0.1 \mathrm{mM}$ of the R-Lim, Eth, Iso, Ben, and Ace binding solutions was faster than that at $1 \mathrm{mM}$. When the concentration is higher, the velocities were slower. These results demonstrate that the frequency equation of the Cp-Lip1 SAW device is effective. There were no significant differences between the experimental and theoretical velocities of Eth, Iso, Ben, and Ace. Remarkably, the experimental velocity of R-Lim was slower than the theoretical velocity, but the difference was not significant. The results may be related to the internal structure of the odorant molecule solutions. Our study provides considerable insight into the Cp-Lip1 SAW device system. These results offer invaluable evidence for the biological function-oriented odor sensors with surface acoustic waves and hydrophobic odorant molecules.

\section{Discussion and Conclusions}

One of the main goals of this study was to develop a method for predicting the Cp-Lip1 odorant molecule protein velocity in the Cp-Lip1 SAW device. Our data suggest that the propagation velocity of the surface acoustic waves is largely related to the liquid density and concentration. We initially thought that the density of the odorant molecules was equal to that of the Cp-Lip1 odorant molecules. However, a more careful analysis inspection revealed that total density of the odorant medium affects all experimental results. These findings thus need to be interpreted carefully.

Our results did not fully meet our expectations. We are aware that our study may have two limitations. The first is the necessity to define the total density of the Cp-Lip1 odorant molecules. The second is that the measured odorant molecules need to have the same concentration. These limitations are evidence of the difficulty in theoretical calculations and experimental data collection. Although our research is based on limited concentrations of the Cp-Lip1 odorant mixture, the final results indicate that the Cp-Lip1 SAW device system can identify different odorant molecules. Our study provides further evidence for SAW theory of biological function-oriented odor sensor research.

In this paper, we presented a SAW theoretical study of the Cp-Lip1 SAW device system. We determined a new mathematical formula for estimating the velocity of SAWs, which propagates in the Cp-Lip1 SAW device. The progress in our new methods is that in the new method, we have taken the property of liquids into consideration, such as density, concentrations, and elastic, which efficiently improve refinement of the calculation of SAWs velocity. We found that the theoretical and experimental propagation velocities of the Cp-Lip1 odorant molecules were nearly identical. We obtained satisfactory results, proving that our frequency equation can predict different odorant molecules in the Cp-Lip1 SAW device. We hope that our research will be beneficial in solving the issues related to biological function-oriented odor sensors. Furthermore, we believe that odorant transfer can be resolved by using this Cp-Lip1 SAW device system. Our study provides the basis for a new method for developing biological function-oriented odor sensors.

Our research suggests that the concentration of odorant molecules has a significant effect on the identification of different odors. We hope that our theoretical calculations will not be affected by odorant concentrations in the future. Surprisingly, for the same odorant molecule, the propagation velocity decreases with increasing concentration. This is a vital issue for future research. Our results are promising and should be validated by a larger sample solution.

\section{Conflicts of Interest}

The authors declare that they have no conflicts of interest.

\section{Acknowledgments}

This work was supported in part by a Grant-in-Aid for Scientific Research (c) (16K07431, 25440164) from Japan Society for the Promotion of Science (JSPS) and a Grant-in-Aid for Scientific Research on Innovative Areas (research in a proposed research area, 24120006) to Tatsuo Iwasa. The authors also thank Dr. Kengo Ogawa, Fine Crystal Co. Ltd., Japan, for his provided experimental data and valuable suggestions. 


\section{References}

[1] K. Toko, "Biomimetic sensor technology," Measurement Science and Technology, vol. 12, no. 2, p. 221, 2001.

[2] C. Caliendo, P. Verardi, E. Verona et al., "Advances in sawbased gas sensors," Smart Materials and Structures, vol. 6, no. 6, pp. 689-699, 1997.

[3] C. Mah and K. B. Thurbide, "Acoustic methods of detection in gas chromatography," Journal of Separation Science, vol. 29, no. 12, pp. 1922-1930, 2006.

[4] L. Kerstin, E. Rapp Bastian, and R. Michael, "Surface acoustic wave biosensors: a review," Analytical and Bioanalytical Chemistry, vol. 391, no. 5, pp. 1509-1519, 2008.

[5] K. Ogawa, T. Torigoe, K. Sawada et al., "Development of the Rayleigh type surface acoustic wave sensor liquid phase system using a longitudinal wave radiation into a liquid phase," IEEJ Transactions on Sensors and Micromachines, vol. 135, no. 12, pp. 490-495, 2015.

[6] V. A. Belogol'skii, S. S. Sekoyan, L. M. Samorukova, S. R. Stefanov, and V. I. Levtsov, "Pressure dependence of the sound velocity in distilled water," Measurement Techniques, vol. 42, no. 4, pp. 406-413, 1999.

[7] D. R. Flower, "Beyond the superfamily: the lipocalin receptors," Biochimica et Biophysica Acta-Protein Structure and Molecular Enzymology, vol. 1482, no. 1-2, pp. 327-336, 2000.

[8] U. Pace, E. Hanski, Y. Salomon, and D. Lancet, "Odorantsensitive adenylate cyclase may mediate olfactory reception," Nature, vol. 316, no. 6025, pp. 255-258, 1985.

[9] T. Nakamura and G. H. Gold, "A cyclic nucleotide-gated conductance in olfactory receptor cilia," Nature, vol. 325, no. 6103, pp. 442-444, 1987.

[10] D. T. Jones and R. R. Reed, "Golf: an olfactory neuron specificG protein involved in odorant signal transduction," Science, vol. 244, no. 4906, pp. 790-795, 1989.

[11] T. Iwasa, G. Mandula, K. Urano et al., "Lipocalin family proteins expressed in the Bowman's gland of the newt olfactory organ," The Japanese Journal of Taste and Smell Research, vol. 15, no. 2, pp. 211-220, 2008.

[12] M. Yamaguchi, B. J. Baer, A. V. Krukau, M. J. McIntire, C. M. Flannery, and E. L. Chronister, "An impulsive stimulated scattering study of surface acoustic waves under high pressure," Journal of Luminescence, vol. 107, no. 1-4, pp. 359368, 2004.

[13] S. Wagle and H. Kato, "Depth evaluation of fretting fatigue crack appearing at bolt joints of aluminum alloy plates by synchronized surface acoustic wave measurement," Materials Transactions, vol. 53, no. 2, pp. 256-263, 2012.

[14] A. Palla-Papavlu, A. Patrascioiu, F. Di Pietrantonio et al., "Preparation of surface acoustic wave odor sensors by laserinduced forward transfer," Sensors and Actuators B: Chemical, vol. 192, pp. 369-377, 2014.

[15] H. Wohltjen and R. Dessy, "Surface acoustic-wave probe for chemical-analysis," Analytical Chemistry, vol. 51, no. 9, pp. 1458-1464, 1979.

[16] H. Wohltjen and R. Dessy, "Surface acoustic-wave probe for chemical-analysis," Analytical Chemistry, vol. 51, no. 9, pp. 1465-1470, 1979.

[17] H. Wohltjen and R. Dessy, "Surface acoustic-wave probe for chemical-analysis," Analytical Chemistry, vol. 51, no. 9, pp. 1470-1475, 1979.
[18] L. Cheol-Min, K. J. Young, and C. Won-Ju, "Field-effect transistor biosensor platform fused with Drosophila odorantbinding proteins for instant ethanol detection," ACS Applied Materials \& Interfaces, vol. 9, no. 16, pp. 14051-14057, 2017.

[19] S. Sindhuja, R. Khot Lav, and P. Suranjan, "Biology and applications of olfactory sensing system: A review," Sensors and Actuators B: Chemical, vol. 171-172, pp. 1-17, 2012.

[20] S. Sindhuja, P. Suranjan, and M. Sanku, "Odorant binding protein based biomimetic sensors for detection of alcohols associated with Salmonella contamination in packaged beef," Biosensors and Bioelectronics, vol. 26, no. 7, pp. 31033109, 2011.

[21] F. Di Pietrantonio, M. Benetti, V. Dinca et al., "Tailoring odorant-binding protein coatings characteristics for surface acoustic wave biosensor development," Applied Surface Science, vol. 302, pp. 250-255, 2014.

[22] M. Redwood, Mechanical Waveguides, Pergamon press, Oxford London New York Paris, 1960.

[23] I. A. Viktorov, Rayleigh and Lame Waves, Plenum press, New York, 1967.

[24] X. Li, K. S. Wendurige, and T. Iwasa, "The study on the structural changes and ligand binding of cp-lip1," The Japanese Journal of Taste and Smell Research, vol. 21, no. 3, pp. 415418, 2014.

[25] Y. Fan, A. N. Sinclair, and F. Honarvar, "Scattering of a plane acoustic wave from a transversely isotropic cylinder encased in a solid elastic medium," The Journal of the Acoustical Society of America, vol. 106, no. 3, pp. 1229-1236, 1999.

[26] N. F. Declercq, J. Degrieck, and O. Leroy, "Diffraction of complex harmonic plane waves and the stimulation of transient leaky Rayleigh waves," Journal of Applied Physics, vol. 98, no. 11, article 113521, 2005. 


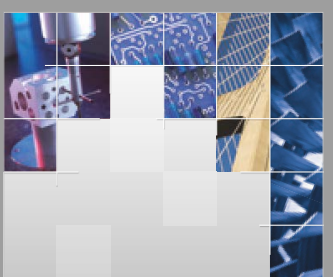

\section{Enfincering}
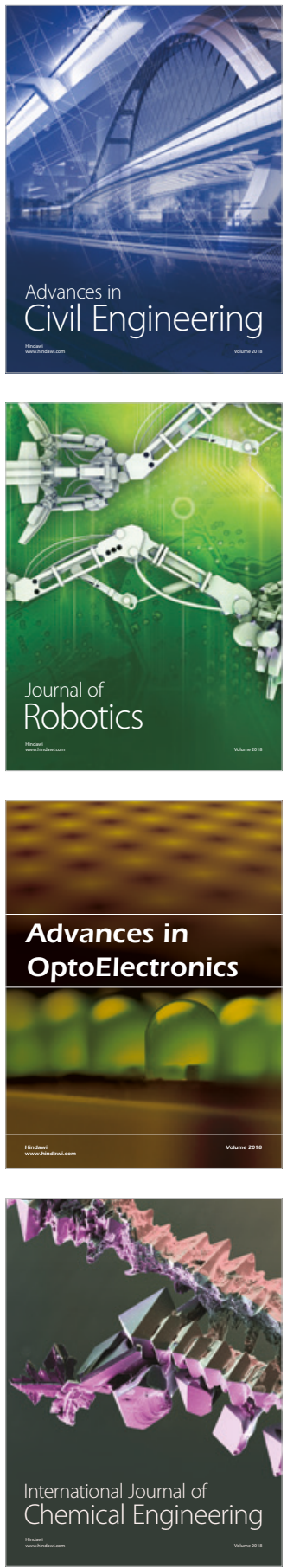

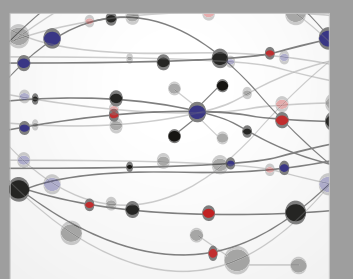

\section{Rotating \\ Machinery}

The Scientific World Journal

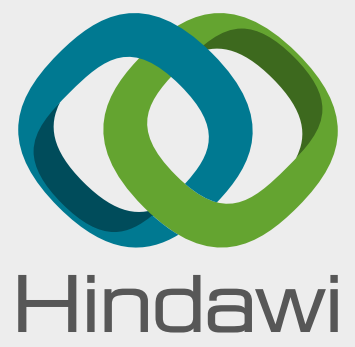

Submit your manuscripts at

www.hindawi.com
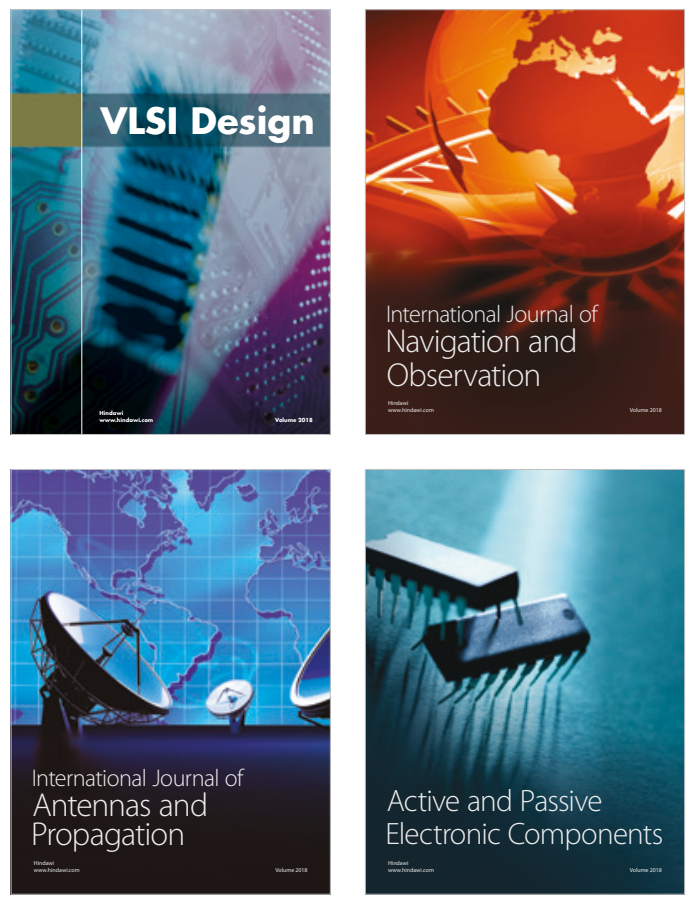
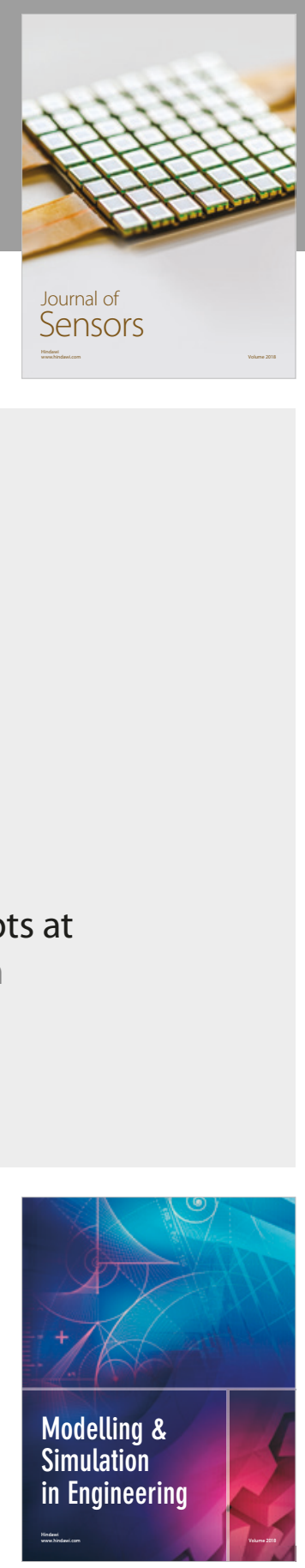

\section{Advances \\ Multimedia}
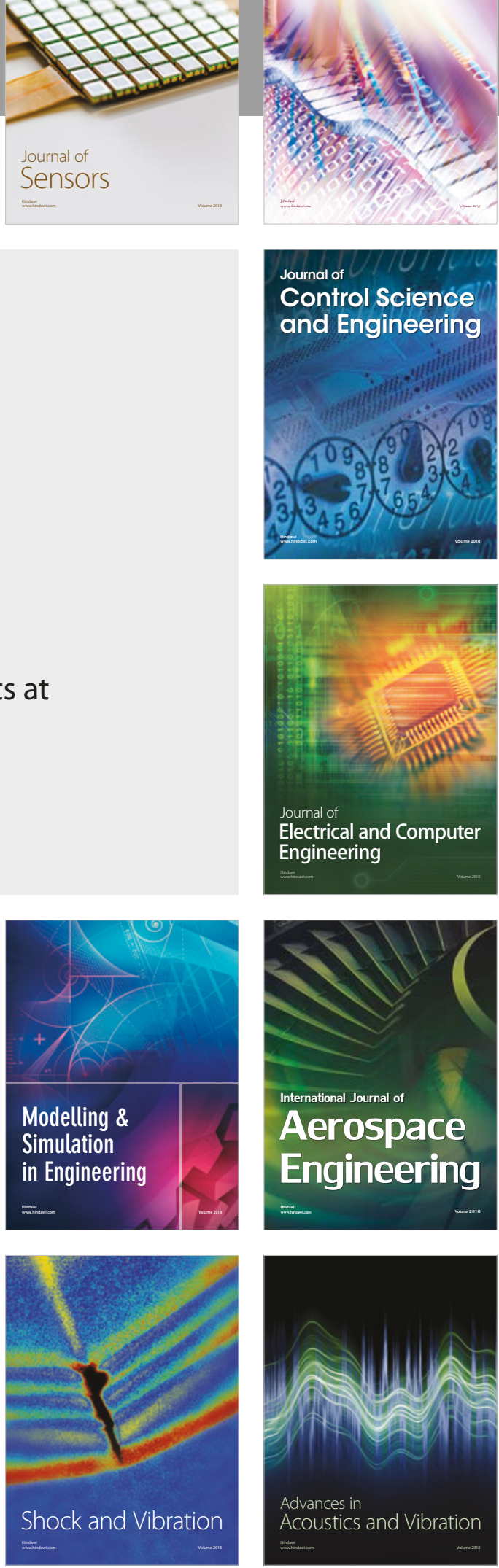